\title{
EP-173
}

\section{Acute pancreatitis disease in the patient with COVID-19 infection: A systematic review}

\author{
$\underline{\text { Ramlah RAMLAH }}^{*}$ \\ Department of Biology, Universitas Gadjah Mada, Indonesia
}

Introduction: The coronavirus disease 2019 (COVID-19) caused by severe acute respiratory syndrome coronavirus 2 (SARS-CoV-2) has become a major outbreak in the world. Few reports have associated COVID-19 with pancreatitis. Angiotensin-converting enzyme-2, the functional virus host cell receptor expressed by organs and tissues, seems to have an important role in the pathophysiology and presentation of this disease. In pancreas, this receptor is expressed in both exocrine glands and islets, being a potential target for the virus and subsequent pancreatic injury. The aim of this study is to determine the relationship between COVID-19 and acute pancreatitis.

Methods: Data obtained from secondary data on 21 articles journal evaluated by searching in PubMed, EMBASE, and the Cochrane Library database that have been carried out in the last 5 years (2016-2020).

Results: The severe COVID-19 pneumonia can lead to pancreatitis. Elevated pancreatic enzymes levels and computed tomography findings led to the diagnosis of acute pancreatitis due to COVID-19. Other research shows that, two of the three family members were diagnosed with acute pancreatitis associated with SARS-CoV-2. Other causes of acute pancreatitis were excluded for both patients (including alcohol, biliary obstruction/gall stones, drugs, trauma, hypertriglyceridemia, hypercalcemia, and hypotension). However, SARS-CoV-2-mediated pancreatic injury had been evident but COVID-19 induced established acute pancreatitis case has seldomly been reported till date globally.

Conclusions: It can be concluded that in acute pancreatitis can occur in some COVID-19 patients. Diagnosing acute pancreatitis secondary to SARS-CoV-2 infection is challenging due to the need to rule out other etiologies as well the notable heterogeneous presentations. 